\title{
Prognostic Value of Heterotypic Neutrophil-in- Tumor Structure in Tongue Squamous Cell Carcinoma Patients
}

Jie Fan ( $\nabla$ zlyyfanjie4235@zzu.edu.cn )

Henan Cancer Hospital https://orcid.org/0000-0003-2316-6284

\section{Yang Yang}

Zhengzhou University First Affiliated Hospital

\section{Qigen Fang}

Henan Cancer Hospital

\section{Meng Cui}

Henan Cancer Hospital

Wei Du

Henan Cancer Hospital

Shanting Liu

Henan Cancer Hospital

\section{Ruihua Luo}

Henan Cancer Hospital

\section{Research}

Keywords: frequency of heterotypic neutrophil-in-tumor structure, tongue squamous cell carcinoma, prognosis, disease-specific survival, tumor grade

Posted Date: December 17th, 2020

DOl: https://doi.org/10.21203/rs.3.rs-127799/v1

License: (a) (1) This work is licensed under a Creative Commons Attribution 4.0 International License. Read Full License 


\section{Abstract}

Objective: To analyze the prognostic value of frequency of heterotypic neutrophil-in-tumor structure(FNiT) in patients with tongue squamous cell carcinoma(TSCC).

Methods: In vitro, we cocultured TSCC cell line-CAL33 with neutrophils to form heterotypic neutrophil-intumor structures, which were then subject to fluorescence staining. Clinically, 197 patients were enrolled. Information including age, sex, FNiT, ECOG PS(Performance Status), FH (family history) of cancer, complications, and pathological characteristics such as tumor stage, node stage, metastasis, disease stage, lymphovascular invasion, perineural invasion, tumor grade, and follow-up results was extracted and analyzed.

Results: Fluorescent staining results of typical heterotypic neutrophil-in-tumor structure showed that welldifferentiated CAL-33-2 had stronger ability to internalize more neutrophils than poorly-differentiated CAL33-1 did, the latter often internalizing only one neutrophil. The mean FNiT was $4.8 \%$,with a range from $2.1 \%$ o to $8.9 \%$ o. The FNiT was significantly associated with tumor stage, disease stage and tumor grade. A total of 119 patients died of the disease, and the 5 -year disease-specific survival(DSS) rate was $36 \%$. The median survival time was 52.6 months. In patients with an FNiT<4.8\%o, the 5-year DSS rate was $40 \%$; in patients with an FNiT $>=4.8 \%$, the 5 -year DSS was $28 \%$, and the difference was significant $(P=0.001)$. Cox model analysis showed that $F N i T$ along with disease stage, lymphovascular invasion and tumor grade was an independent prognostic factor for DSS.

Conclusion: The FNiT as a novel predictor is positively correlated with adverse prognosis of patients with TSCC.

\section{Introduction}

Cell cannibalism is an evolutionarily conservative cytobiological phenomenon(1), which has been noted in varieties of solid tumors including head and neck squamous cell carcinoma(HNSCC)(2).Cannibalistic cells generally form cell-in-cell structures, for which one living cell is internalized into another one $(3,4)$. Cells of different origin form heterotypic cell-in-cell structures, which often occurs between tumor cells and immune cells with neutrophils included(5). Neutrophils are the most important marker and trigger factor of inflammation. Recent reseaches have imply that inflammation along with metabolic and immune response to stimuli from the surrounding tumor tissues constitutes the tumor microenvironment, which signifcantly affects cancer progression through interacting with tumor cells(6). Neutrophils may significantly impact on the tumor microenvironment through chemokines and cytokines that result in imflammatory cell recruitment and activation, which could promote tumor cell proliferation, microvascular regeneration and tumor metastasis $(7,8)$.

Neutrophil-in-tumor structure is a typical heterotypic cell-in-cell structure, which has been reported in HNSCC. Frequency of neutrophil-in-tumor structure formation(FNiT) reflects the severity of tumorinfiltrating neutrophils and is calculated from the number of neutrophil-in-tumor structure divided by the 
total number of tumor cells. Several experts have found that neutrophil-in-tumor structure formation is associated with adverse prognosis in patients with $\operatorname{HNSCC}(2,5,9)$. However, whether the FNiT affects prognosis in patients with TSCC(TSCC) is still unclear. Therefore, the current study aimed to uncover the mystery.

\section{Materials And Methods}

\section{Patients and methods}

The Institutional Research Committee of Henan Cancer Hospital approved our study. All participants signed an informed consent agreement for medical research before initial treatment, and all experiments were performed in accordance with relevant guidelines and regulations. The study was conducted in accordance with the Declaration of Helsinki and all subjects provided informed consent (see Attachment 1 for details).

From February 2010 to September 2019, the surgical medical records of all patients(>29 years old) diagnosed with TSCC were retrospectively included. All related data, including age, sex, FNiT, ECOG PS, FH of cancer, complications, tumor stage, node stage, metastasis, disease stage, lymphovascular invasion, perineural invasion, tumor grade, postoperative pathological report, operation record, adjuvant treatment and follow-up information, of the enrolled patients were extracted and analyzed. All pathologic sections were re-reviewed. The tumor grade was defined according to the WHO 2017 classification, and the tumor stage was defined based on the AJCC eighth Edition staging system. In our cancer center, preoperative ultrasound and CT or MRI were routinely performed. In addition, frozen sectioning of the primary tumor was routinely performed; if the pathology was malignant, a total glossectomy was performed.

The FNiT was defined as the frequency of heterotypic cell-in-cell(neutrophils inside tumor cells) formation in the pathologic sections of TSCC. The cutoff value calculated from the ROC curve, mean, tertile, or median in previous studies varied. Thus, the standard cutoff value remains unknown. In the current study, the cutoff value was defined as the mean value of the FNiT.

The chi-squared test was used to assess the association between the FNiT and the clinicopathologic variables. The Kaplan-Meier method was used to calculated the disease-specific survival(DSS) rate. The Cox proportional hazards method was used to determine the independent risk factors for DSS. All statistical analyses were conducted with the help of SPSS version 20(IBM Corporation, Armonk, NY, USA). A P $<0.05$ was considered significant.

\section{Cells And Culture Conditions}

CAL-33 cells were maintained in Dulbecco's modified Eagle's medium supplemented with $10 \%$ fetal bovine serum(PAN-Biotech). About $1 \times 10^{5}$ CAL-33 cells were adherently cultured in 12-well plates. 
Neutrophils were maintained in RPMI-1640 supplemented with 10\% fetal bovine serum(PAN-Biotech). About $1 \times 10^{6}$ neutrophils were cultured in suspension in $10-\mathrm{cm}$ dish.

\section{Heterotypic Cic Formation Assay}

Briefly, about $1 \times 10^{5} \mathrm{CAL}-33$ cells were adherently cultured in 12-well plates for $8 \mathrm{~h}$. Then coculture neutrophils and CAL-33 cells for $8 \mathrm{~h}$. Cytospins were then made by centrifugation at $800 \mathrm{rpm}$ for $4 \mathrm{~min}$. And cells were then fixed and stained with phalloidin-568 and Hoechst to quantify hClC structures. Internalized cells wrapped by outer cells were considered as hCICs.

\section{Fluorescence Staining}

Firstly, neutrophils were stained with CellTracker Green(Invitrogen); Secondly, cytospins were fixed in 4\% paraformaldehyde and then proceeded to routine staining with phalloidin-568(Life) and Hoechst(Thermo) for 30 min before mounted with Prolong Gold antifade reagent (Invitrogen). Confocal images were captured and processed by Ultraview Vox confocal system (Perkin Elmer) on Nikon Ti-E microscope.

\section{Results}

In vitro, we cocultured TSCC cell line-CAL33 with neutrophils to form neutrophil-in-tumor strutures. Fluorescent staining results of typical heterotypic neutrophil-in-tumor structure were shown in Fig. 1. CAL33-1 and CAL-33-2 are poorly-differentiated and well-differentiated TSCC cell lines, respectively. Cells marked in red and green are CAL-33 and neutrophils, respectively. The region marked in blue is the nuclei of CAL-33 and neutrophils. We discovered that well-differentiated CAL-33-2 had stronger ability to internalize more neutrophils than poorly-differentiated CAL-33-1 did, the latter often internalizing only one neutrophil.

Clinically, in total, 197 patients(93 females and 104 males) were enrolled with a mean age of 56.6(range: 29-87) years. FNiT > $=4.8 \%$ o was detected in $91(46.2 \%)$ patients, while FNiT < 4.8\%o was in 106(53.8\%) patients. All patients were measured as ECOG Performance Status(ECOG PS) 1 level. Family history of cancer(FH of cancer) was found in $10(5.1 \%)$ patients. Complications were noted in 63(32.0\%) patients. Tumor stage was distributed as follows: T1 in 62(31.5\%) patients, T2 in 37(18.8\%) patients, T3 in $71(36.0 \%)$ patients, and T4 in 27(13.7\%) patients. Distant metastasis was noted in 16(8.1\%) patients. Lymphovascular invasion and perineural invasion were noted in $15(7.6 \%)$ and $19(9.6 \%)$ patients, respectively. Tumor grade was distributed as follows: low grade in $13(6.6 \%)$ patients, median grade in $39(19.8 \%)$ patients, high grade in $145(73.6 \%)$ patients(Table 1). A negative margin was achieved in $189(96.0 \%)$ patients. The mean FNiT was $4.8 \%$, with a range from $2.1 \%$ o to $8.9 \%$ o. 
Table 1

Association between FNiT and clinicopathological characteristics

\begin{tabular}{|c|c|c|c|}
\hline Variables & FNiT & & P-value \\
\hline & $\operatorname{Low}(<4.8 \%$ o $)$ & $\operatorname{High}(>=4.8 \%$ o & \\
\hline & $n=106$ & $n=91$ & \\
\hline Age(years) & & & 0.684 \\
\hline$<50$ & 52 & 42 & \\
\hline$\geq 50$ & 54 & 49 & \\
\hline Sex & & & 0.156 \\
\hline Male & 51 & 53 & \\
\hline Female & 55 & 38 & \\
\hline ECOG PS & & & N/A \\
\hline 0 & & & \\
\hline 1 & 106 & 91 & \\
\hline 2 & & & \\
\hline FH of cancer & & & 0.687 \\
\hline Y & 6 & 4 & \\
\hline$N$ & 100 & 87 & \\
\hline Complications & & & 0.849 \\
\hline Y & 35 & 28 & \\
\hline$N$ & 71 & 63 & \\
\hline Tumor stage & & & 0.027 \\
\hline $\mathrm{T} 1+\mathrm{T} 2$ & 61 & 38 & \\
\hline $\mathrm{T} 3+\mathrm{T} 4$ & 45 & 53 & \\
\hline Node stage & & & 0.485 \\
\hline NO & 88 & 72 & \\
\hline $\mathrm{N}+$ & 18 & 19 & \\
\hline Metastasis & & & 0.400 \\
\hline Positive & 7 & 9 & \\
\hline Negative & 99 & 82 & \\
\hline
\end{tabular}




\begin{tabular}{|lccc|}
\hline Variables & FNiT & & P-value \\
\hline Disease stage & 56 & 34 & 0.030 \\
I + II & 50 & 57 & \\
III + IV & & & 0.564 \\
Lymphovascular invasion & & 8 & \\
\hline Positive & 7 & 83 & 0.282 \\
\hline Negative & 99 & & \\
\hline Perineural invasion & & 11 & \\
\hline Positive & 8 & 80 & \\
\hline Negative & 98 & & \\
\hline Tumor Grade & & 3 & \\
\hline Low & 10 & 11 & \\
\hline Median & 28 & 77 & \\
\hline High & 68 & & \\
\hline
\end{tabular}

In total, $160(81.2 \%)$ patients were classified as $\mathrm{cNO}$, and 51 patients underwent neck dissection; positive neck disease was reported in 13 patients. In total, 37(18.8\%) patients were classified as cN+, and underwent neck dissection; positive neck disease was reported in 35 patients.

When analyzing the association between the FNiT and clinicopathological variables, it was noted that the FNiT was significantly associated with tumor grade(Table 1).

During our follow up with a mean time of 54.9(range: 13-115) months, 101 patients had received radiotherapy, 26 patients received adjuvant chemotherapy. A total of 119 patients died of the disease, and the 5 -year DSS rate was $36 \%$. The median survival time was 52.6 months. When analyzing the predictors for DSS, it was noted that a high FNiT was associated with a poor prognosis in patients with an FNiT< $4.8 \%$ o the 5 -year DSS rate was $40 \%$ and in patients with an FNiT > $=4.8 \%$, the 5 -year DSS was $28 \%$; the difference was significant $(P=0.001$, Fig. 2). Further, the Cox model proved that FNiT along with disease stage, lymphovascular invasion and tumor grade was an independent predictor of DSS (Table 2). Disease stage(III + IV) and positive lymphovascular invasion were associated with an adverse prognosis in patients with TSCC, respectively(Fig. 3A and B). Patients with high tumor grade tended to have a shorter survival time than those with low or median tumor grade did(Fig. 3C). 
Table 2

Univariate and multivariate analysis of the predictors for disease-specific survival in patients with TSCC

\begin{tabular}{|c|c|c|c|}
\hline \multirow[t]{2}{*}{ Variables } & \multirow{2}{*}{$\begin{array}{l}\text { Univariate } \\
\text { P-value }\end{array}$} & \multicolumn{2}{|c|}{ Cox model } \\
\hline & & P-value & OR(95\% Cl) \\
\hline Age,years(< 50 vs $\geq 50)$ & 0.815 & & \\
\hline Sex(male vs female) & 0.151 & & \\
\hline FH of cancer(Y vs N) & 0.083 & & \\
\hline Complications(Y vs N) & 0.972 & & \\
\hline FNiT(Low vs High) & 0.001 & 0.017 & $1.569(1.084-2.270)$ \\
\hline Tumor stage(T1 + T2 vs T3 + T4) & 0.003 & 0.140 & $0.501(0.200-1.254)$ \\
\hline Node stage(N0 vs N+) & 0.288 & & \\
\hline Metastasis(Y vs N) & $<0.001$ & 0.888 & $1.108(0.266-4.623)$ \\
\hline Disease stage(I + II vs III + IV) & $<0.001$ & 0.008 & $3.808(1.417-10.233)$ \\
\hline Lymphovascular invasion( $\mathrm{Y}$ vs $\mathrm{N}$ ) & 0.001 & 0.004 & $2.373(1.315-4.282)$ \\
\hline Perineural invasion(Y vs $\mathrm{N})$ & $<0.001$ & 0.796 & $1.179(0.339-4.096)$ \\
\hline Tumor Grade & 0.004 & 0.001 & $1.822(1.273-2.607)$ \\
\hline \multicolumn{4}{|l|}{ Low } \\
\hline \multicolumn{4}{|l|}{ Median } \\
\hline High & & & \\
\hline
\end{tabular}

\section{Discussion}

Studies indicate that heterotypic cell-in-cell(hClC) structures occur in the penetration of immune cells into tumor cells or thymic nurse cells and others, discovered in the intestine epithelia by a German scholar 150 years ago(10). In tumor tissues, tumor cells are an important target of hClC, and various tumor cells seem to be able to internalize immune cells. For example, HNSCC, melanoma, gastric cancer, ductal carcinoma of salivary gland, breast cancer, liver cancer and other tumor cells $(11,12)$. Similarly, a variety of immune cells can enter into tumor cells, such as neutrophils(11-14),NK cells, T lymphocytes, LAK cells, B cells et al., among which neutrophils were mostly reported. hClC structure is closely related to tumor, which is not only common in various tumor tissues, but also significantly correlated with the pathological grade of various tumors, suggesting that the formation of hClC structure may play an important role in the occurrence and development of tumor. In addition, studies in Jerry's laboratory have shown that thymic nurse cells can internalize the developing $\mathrm{T}$ cells to form the $\mathrm{hClC}$ structure to prevent 
T cells from apoptosis, and this process is also involved in the negative and positive selection of $\mathrm{T}$ cells by thymic nurse cells(15), which indicates that hCIC plays an important role in the development and maturation of T cells.

Heterotypic neutrophil-in-tumor structures formed by neutrophils penetrating into tumor cells are often noted in the solid tumors with HNSCC included $(16,17)$. In our study, we cocultured TSCC cell line-CAL33 with neutrophils in vitro to form neutrophil-in-tumor strutures. We found that well-differentiated tumor cells have higher ability to internalize more neutrophils than poorly-differentiated tumor cells. Though in the association between clinical pathologic characteristics and FNiT we noted that tumor grade was positively correlated with FNiT, and well-differentiated tumor tended to have low FNiT. That is, frequency of neutrophil-in-tumor structures in well-differentiated tumor tissue is lower than that of poorlydifferentiated tumor tissue. This is not contradictory to the result of heterotypic cell-in-cell assay in vitro mentioned above. We speculate that the tumor tissues with low FNiT have less neutrophils infiltration than the ones with high FNiT, but the proportion of well-differentiated tumor cells in the former outcompeted that in the latter. This is consistent with the outcome of the patients between with low FNiT and with high FNiT mentioned previously. We could further infer that tumors with more neutrophils infiltration have an adverse prognosis than tumors with less neutrophils infiltration.

The value of FNiT in the prognosis of patients with TSCC has scarcely been investigated to date. A few of papers have reported the existence of neutrophil-in-tumor structures in other tumors of HNSCC. Seza Tetikkurt et al(9) reported a case depicting the presence of the significant neutrophil-in-tumor structures in the squamous cell carcinoma of the hard palate and maxilla which is rarely encountered. Neutrophil-intumor structures may predict tumor behavior and the consequences of immune-modulating treatment response in HNSCC. S. C. Sarode et al(16) revealed neutrophil-in-tumor structrues were significantly correlated with poor differentiation of tumor cells and cervical lymph node metastasis. Similar findings of the relationship between FNiT and tumor grade were also noted in current study. Gupta K et al(1) found that cell-in-cell structure was a dependable cytological feature of malignancy. This implied that high FNiT was positively correlated with the malignancy and relevant adverse outcomes of tumors, which was consistent with our views mentioned previously. Furthermore, neutrophil-in-tumor structures have been identified by light microscopy evaluation of pleomorphic cell (giant cell) carcinomas of the intestine, lung, and pancreas, breast carcinomas, invasive micropapillary carcinomas of the ampulopancreaticobiliary region, gastric carcinomas, and lymphomas $(11,14,18-21)$.

In our study, we innovatively explored the value of FNiT in the prognosis of patients with TSCC and found that FNiT is significantly associated with DSS. However, the mechanism underlying the association between prognosis as well as clinicopathological characteristics remains unclear. As we proposed above, the FNiT reflects the degree of neutrophil infiltration within the tumor microenvironment. Neutrophils may lead to a significant impact on the tumor microenvironment through cytokines and chemokines that cause inflammatory cell recruitment and activation which could promote tumor cell proliferation, microvascular regeneration and tumor metastasis as a result(7-9). This may better explain the reason why patients with high FNiT tended to have lower 5-year DSS rate than those with low FNiT. The specific 
role of neutrophils in the pathogenesis of cancer has recently become the subject of research with special focus on the association between tumor microenvironment and tumor progression. Neutrophils may play a crucial role in cancer biology and may act as tumor promotor in tumor progression. Neutrophils may be vital biomarkers for HNSCC and targets to control the progression of $\operatorname{HNSCC}(8,22)$. Mackay HL et al. (23)disclosed that neutrophil-in-tumor structures are positively correlated with poor prognosis in cancer patiets.

In the univariate and multivariate analysis of the predictors for DSS in patients with TSCC, we currently discovered that FNiT, disease stage, lymphovascular invasion and tumor grade were four independent predictors for DSS in the Cox model. However, other candidate predictors such as tumor stage, node stage, metastasis and perineural invasion were not proved to be independently associated with DSS. We did not expect this. Possible speculations may be that the proportions of the positive patient of the node stage, metastasis and perineural invasion parameters were all pretty small, which would have strong error and bias in the survival analysis. Particularly, our data indicated that tumor stage may be correlated with DSS dependent on FNiT. In the research on the association between FNiT and clinicopathological characteristics, we concluded that FNiT was associated with tumor stage $(p=0.027)$.

\section{Limitations}

Limitations of the current study should be acknowledged. First, our study was a retrospective form, so the inherent bias might reduce the statistic power. Second, the clinicopathological parameters were disproportionally distributed in the patients enrolled in our study, which resulted in strong error in the relevant statistical analysis.

\section{Conclusions}

The FNiT as a novel predictor is positively correlated with adverse prognosis of patients with TSCC.

\section{Abbreviations}

FNiT: Frequency of heterotypic neutrophil-in-tumor structure; TSCC: Tongue squamous cell carcinoma; HNSCC: Head and neck squamous cell carcinoma.

\section{Declarations}

\section{Funding statement}

This research was supported by the National Natural Science Foundation of China (8157110152).

\section{Data availability statement}


All data generated or analyzed during this study are included in this published article. The primary data can be obtained from the corresponding author.

\section{Ethics statement}

The studies involving human participants were reviewed and approved by the Institutional Research Committee of Henan Cancer Hospital. The participants provided informed consent to participate in this study(see Attachment 1 for details).

\section{Authors' contributions}

FJ wrote the manuscript; YY collected clinical data; CM performed statistical analysis; FQ and DW performed fluorescence staining assay, and figure preparation; FQ, LS and LR made manuscript preparation suggestions and final manuscript revision. All authors read and approved the final manuscript.

\section{Consent for publication}

Written informed consent was obtained from the patient's legal guardian for publication of this article and any accompanying images. A copy of the written consent (see Attachment 1 for details) is available for review by the Editor-in-Chief of this journal..

\section{Conflict of interest statement}

The authors declare that they have no conflict of interest.

\section{Acknowledgements}

We thank Dr. Zhang He for his instructive help on data analysis.

\section{References}

1. Gupta K, Dey P. Cell cannibalism: diagnostic marker of malignancy. Diagn Cytopathol. 2003;28(2):86-7.

2. Schenker H, Büttner-Herold M, Fietkau R, Distel LV. Cell-in-cell structures are more potent predictors of outcome than senescence or apoptosis in head and neck squamous cell carcinomas. Radiation oncology (London England). 2017;12(1):21-.

3. Fais S, Overholtzer M. Cell-in-cell phenomena in cancer. Nat Rev Cancer. 2018;18(12):758-66.

4. Liang J, Fan J, Wang M, Niu Z, Zhang Z, Yuan L, et al. CDKN2A inhibits formation of homotypic cellin-cell structures. Oncogenesis. 2018;7(6):50-

5. Caruso RA, Fedele F, Finocchiaro G, Arena G, Venuti A. Neutrophil-tumor cell phagocytosis (cannibalism) in human tumors: an update and literature review. Experimental oncology. 
2012;34(3):306-11.

6. Hanahan D, Coussens LM. Accessories to the crime: functions of cells recruited to the tumor microenvironment. Cancer Cell. 2012;21(3):309-22.

7. Baniyash M, Sade-Feldman M, Kanterman J. Chronic inflammation and cancer: suppressing the suppressors. Cancer Immunol Immunother. 2014;63(1):11-20.

8. Gregory AD, Houghton AM. Tumor-associated neutrophils: new targets for cancer therapy. Cancer Res. 2011;71(7):2411-6.

9. Tetikkurt S, Taş F, Emre F, Özsoy Ş, Bilece ZT. Significant Neutrophilic Emperipolesis in Squamous Cell Carcinoma. Case reports in oncological medicine. 2018;2018:1301562-.

10. Overholtzer M, Brugge JS. The cell biology of cell-in-cell structures. Nat Rev Mol Cell Biol. 2008;9(10):796-809.

11. Khayyata S, Basturk O, Adsay NV. Invasive micropapillary carcinomas of the ampullopancreatobiliary region and their association with tumor-infiltrating neutrophils. Modern pathology: an official journal of the United States Canadian Academy of Pathology Inc. 2005;18(11):1504-11.

12. Singhal N, Handa U, Bansal C, Mohan H. Neutrophil phagocytosis by tumor cells-a cytological study. Diagn Cytopathol. 2011;39(8):553-5.

13. Arya P, Khalbuss WE, Monaco SE, Pantanowitz L. Salivary duct carcinoma with striking neutrophiltumor cell cannibalism. Cytojournal. 2011;8:15-

14. Caruso RA, Muda AO, Bersiga A, Rigoli L, Inferrera C. Morphological evidence of neutrophil-tumor cell phagocytosis (cannibalism) in human gastric adenocarcinomas. Ultrastruct Pathol. 2002;26(5):31521.

15. Martinez M, Samms M, Hendrix TM, Adeosun O, Pezzano M, Guyden JC. Thymic nurse cell multicellular complexes in HY-TCR transgenic mice demonstrate their association with MHC restriction. Exp Biol Med (Maywood). 2007;232(6):780-8.

16. Sarode SC, Sarode GS. Neutrophil-tumor cell cannibalism in oral squamous cell carcinoma. Journal of oral pathology medicine: official publication of the International Association of Oral Pathologists the American Academy of Oral Pathology. 2014;43(6):454-8.

17. Saxena S, Beena KR, Bansal A, Bhatnagar A. Emperipolesis in a common breast malignancy. A case report. Acta cytologica. 2002;46(5):883-6.

18. Bak M, Teglbjaerg PS. Pleomorphic (giant cell) carcinoma of the intestine. An immunohistochemical and electron microscopic study. Cancer. 1989;64(12):2557-64.

19. Fishback NF, Travis WD, Moran CA, Guinee DG Jr, McCarthy WF, Koss MN. Pleomorphic (spindle/giant cell) carcinoma of the lung. A clinicopathologic correlation of 78 cases. Cancer. 1994;73(12):2936-45.

20. Gupta RK, Wakefield SJ. Needle aspiration cytology, immunocytochemistry, and electron microscopic study of unusual pancreatic carcinoma with pleomorphic giant cells. Diagn Cytopathol. 1992;8(5):522-7. 
21. Sarode GS, Sarode SC, Patil S. Emperipolesis: An Unreported Novel Phenomenon in Oral Squamous Cell Carcinoma. J Contemp Dent Pract. 2017;18(4):345-7.

22. Magalhaes MAO, Glogauer JE, Glogauer M. Neutrophils and oral squamous cell carcinoma: lessons learned and future directions. J Leukoc Biol. 2014;96(5):695-702.

23. Mackay HL, Muller PAJ. Biological relevance of cell-in-cell in cancers. Biochem Soc Trans. 2019;47(2):725-32.

\section{Figures}

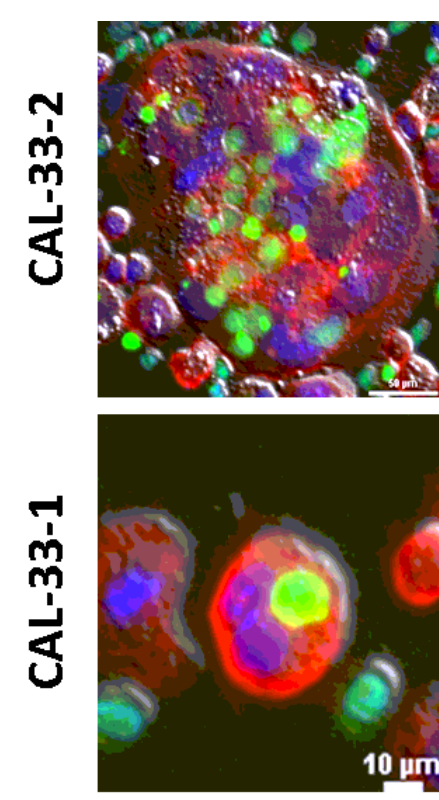

Merge
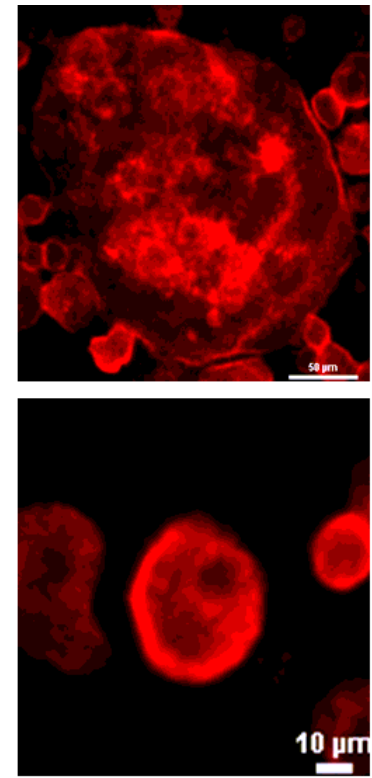

CAL-33
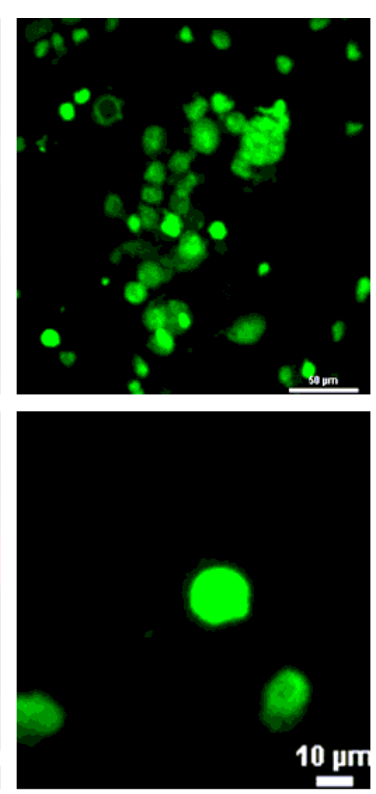

Neutrophil
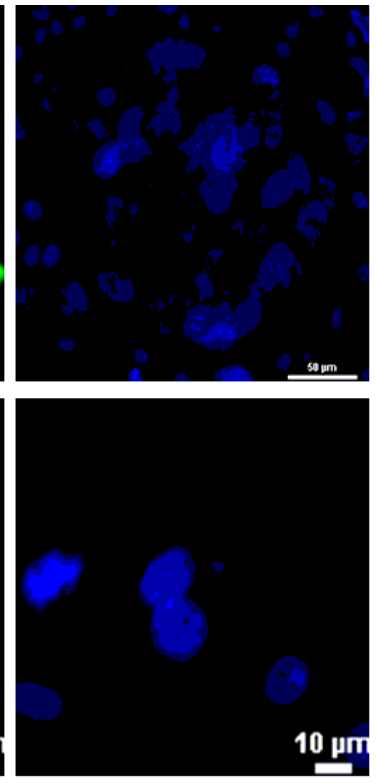

Nuclei
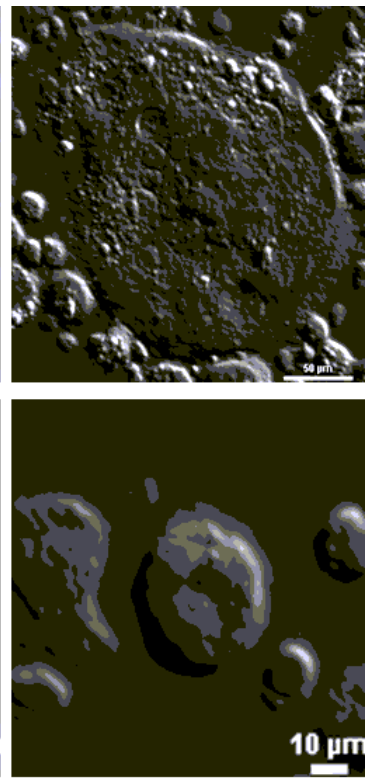

Bright

\section{Figure 1}

Fluorescent staining result of typical heterotypic neutrophil-in-tumor structure formed between CAL-33 and neutrophils. CAL-33-1 and CAL-33-2 are poorly-differentiated and well-differentiated TSCC cell lines, respectively. Cells marked in red and green are CAL-33 and neutrophils, respectively. The region marked in blue is the nuclei of CAL-33 and neutrophils. Scale bar of first row: 50um; scale bar of second row: 10um. 

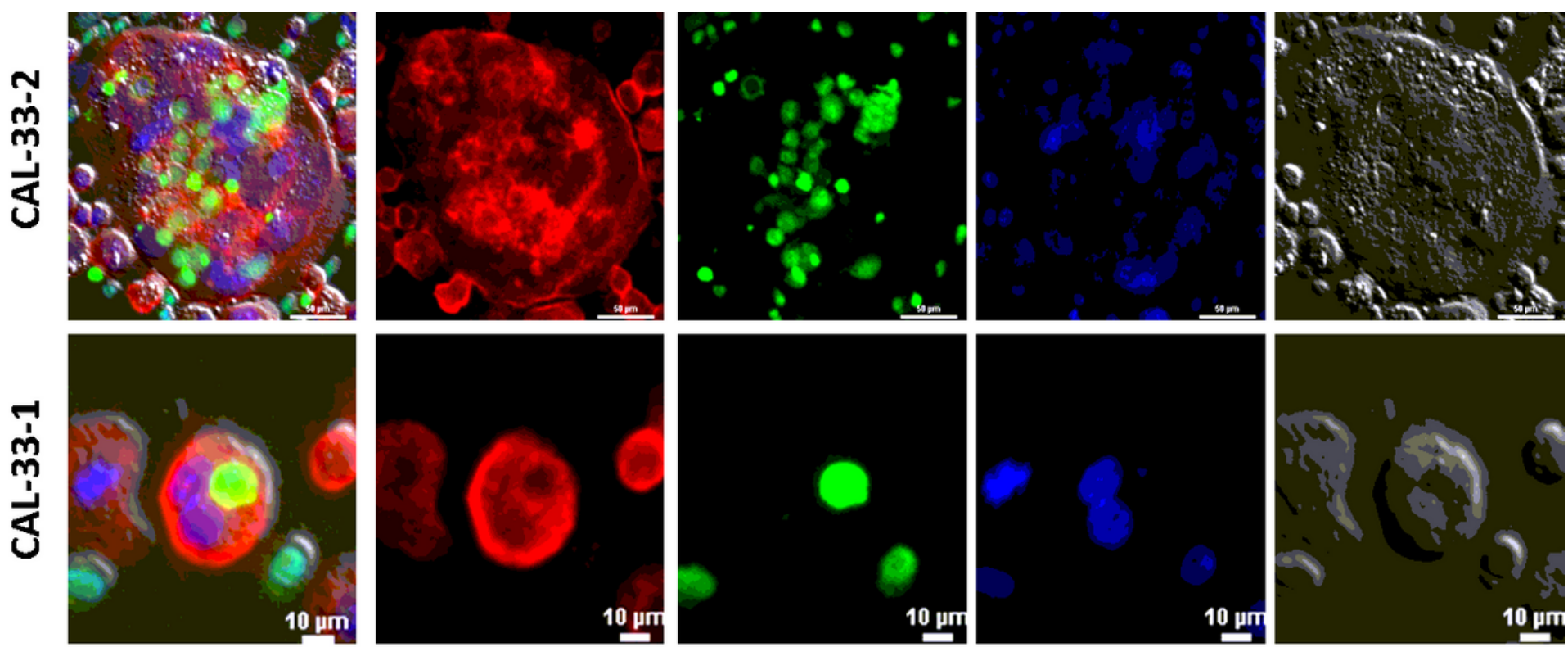

Merge

CAL-33

Neutrophil

Nuclei

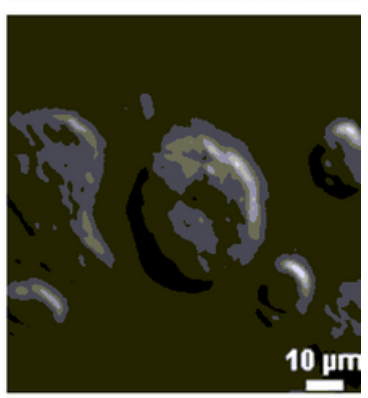

Bright

Figure 1

Fluorescent staining result of typical heterotypic neutrophil-in-tumor structure formed between CAL-33 and neutrophils. CAL-33-1 and CAL-33-2 are poorly-differentiated and well-differentiated TSCC cell lines, respectively. Cells marked in red and green are CAL-33 and neutrophils, respectively. The region marked in blue is the nuclei of CAL-33 and neutrophils. Scale bar of first row: 50um; scale bar of second row: 10um.
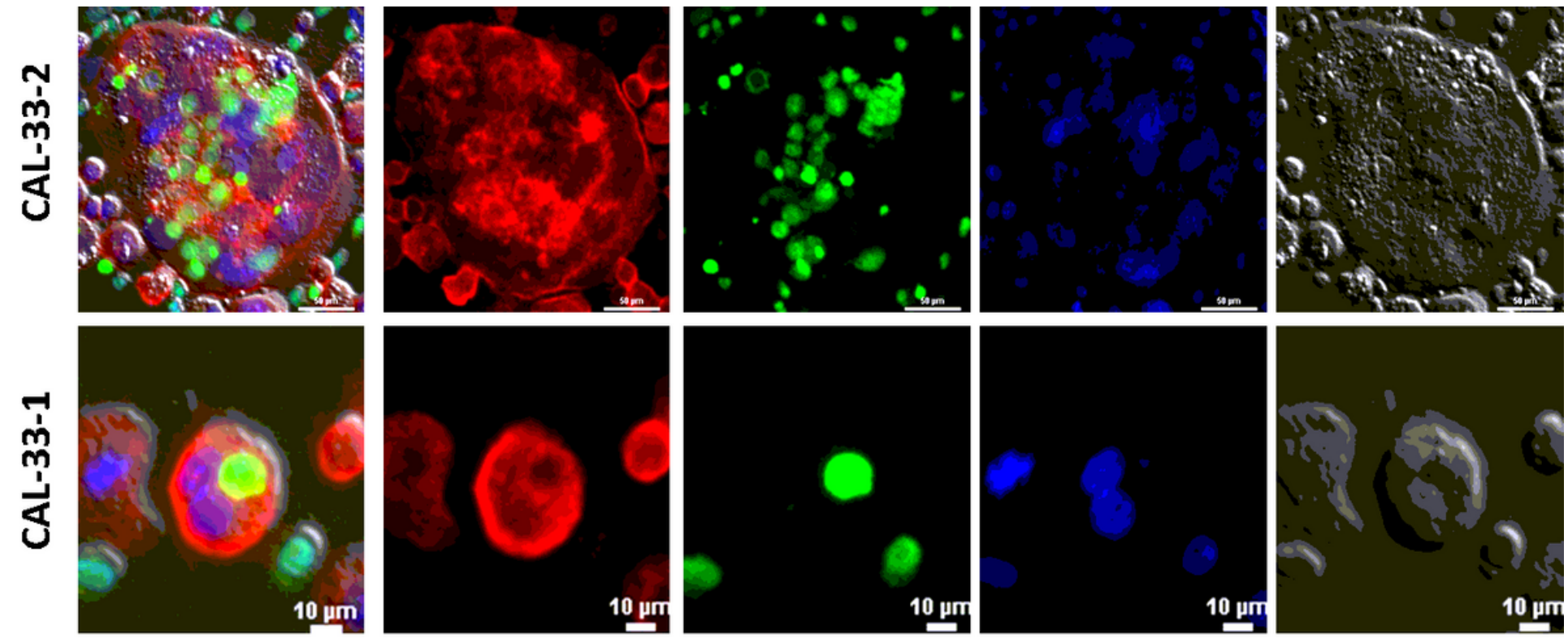

Merge

CAL-33

Neutrophil

Nuclei

Bright

Figure 2

Fluorescent staining result of typical heterotypic neutrophil-in-tumor structure formed between CAL-33 and neutrophils. CAL-33-1 and CAL-33-2 are poorly-differentiated and well-differentiated TSCC cell lines, 
respectively. Cells marked in red and green are CAL-33 and neutrophils, respectively. The region marked in blue is the nuclei of CAL-33 and neutrophils. Scale bar of first row: 50um; scale bar of second row: 10um.

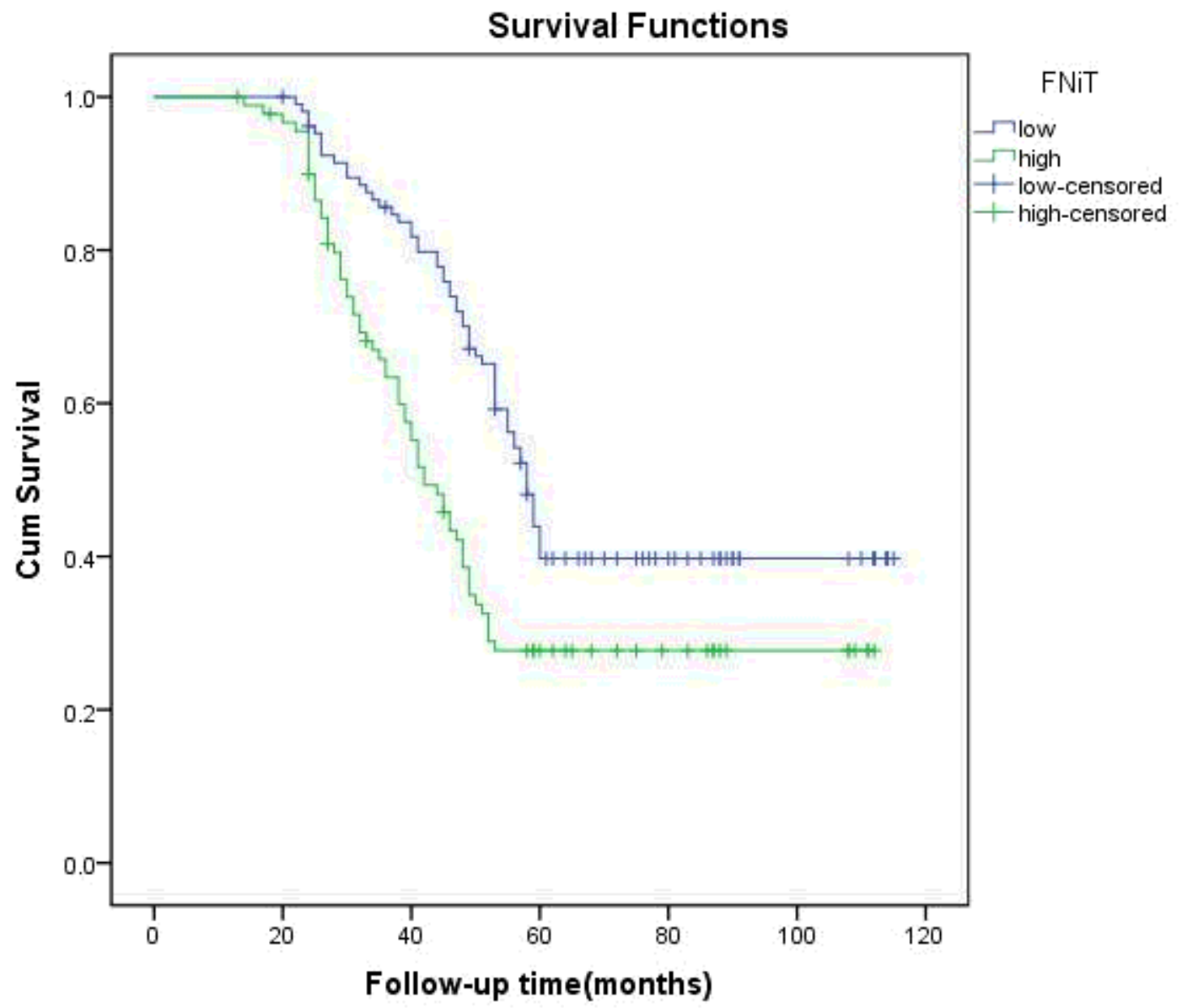

Figure 3

Disease-specific survival function in patients with different FNiT. Note: $p=0.001$. 


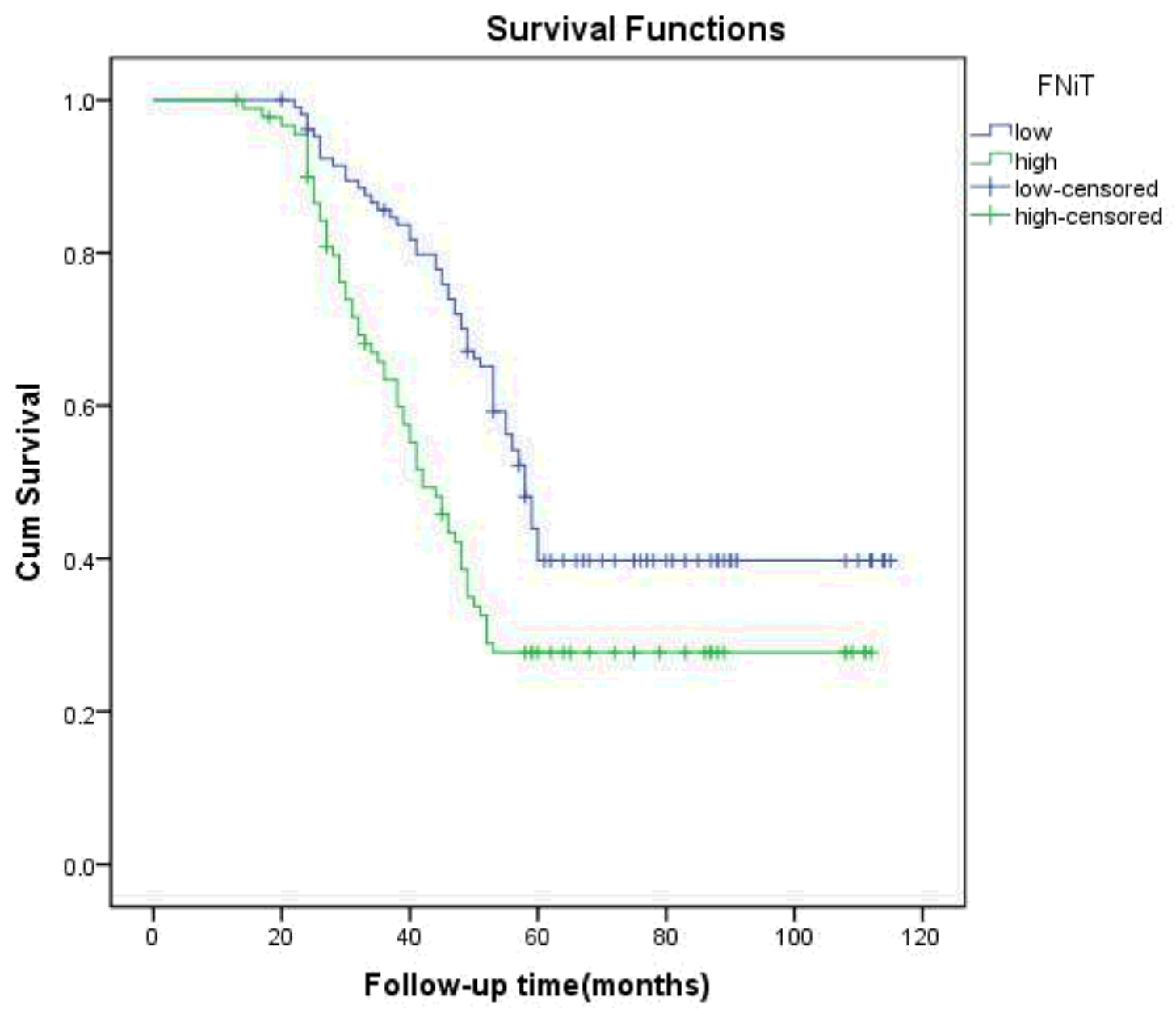

Figure 3

Disease-specific survival function in patients with different FNiT. Note: $p=0.001$. 


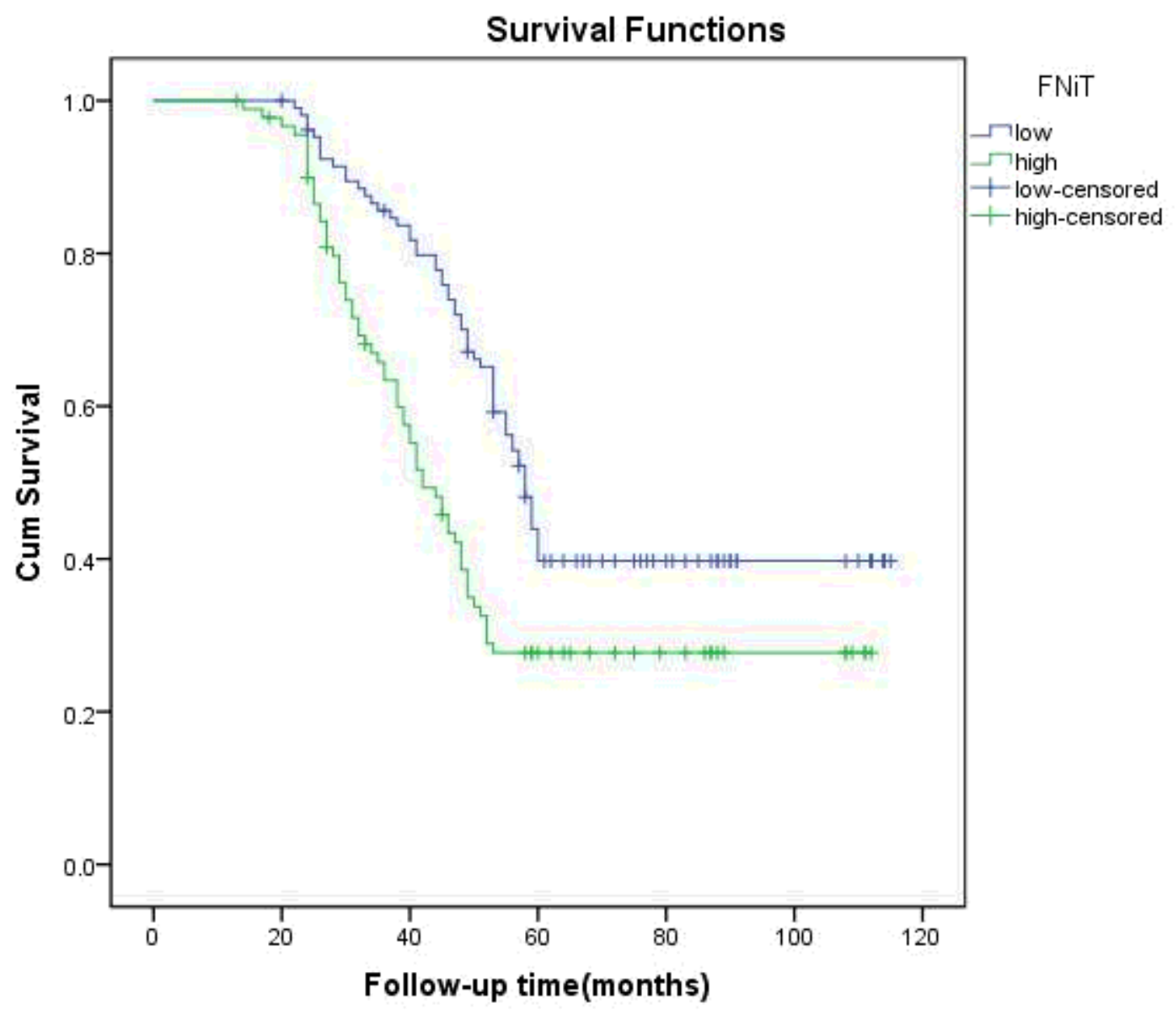

Figure 4

Disease-specific survival function in patients with different FNiT. Note: $p=0.001$. 
A

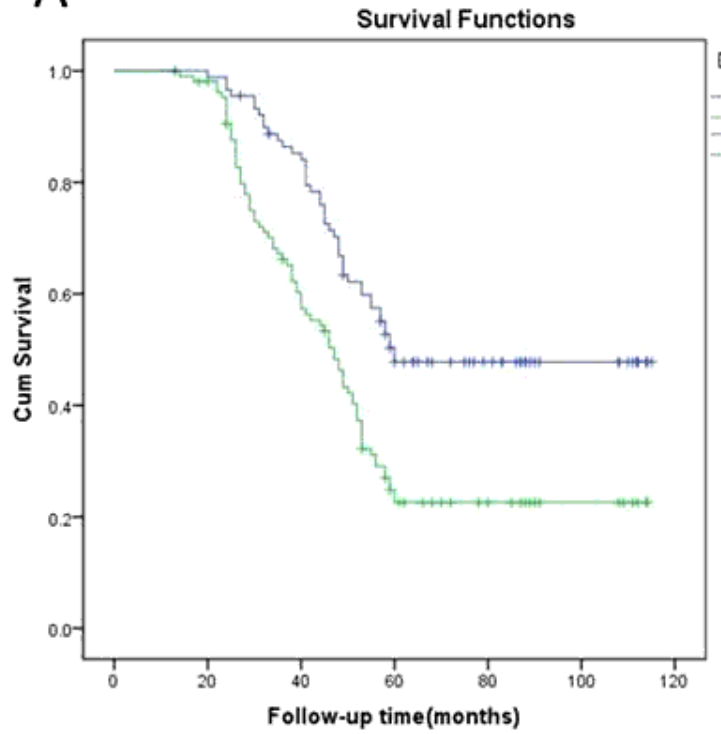

B

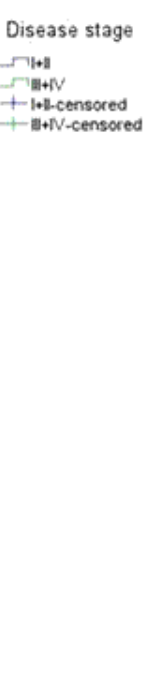

Survival Functions

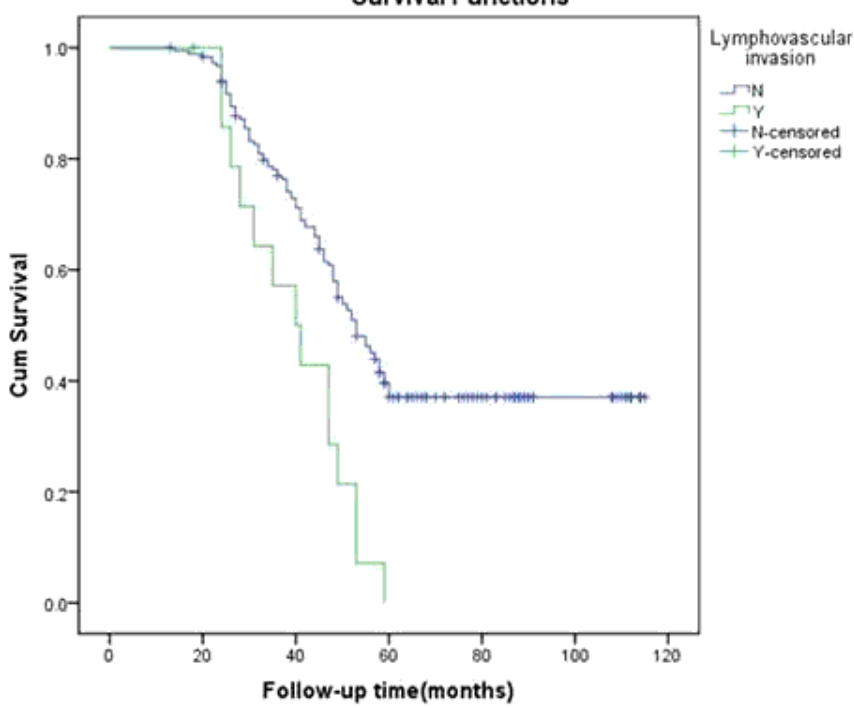

C

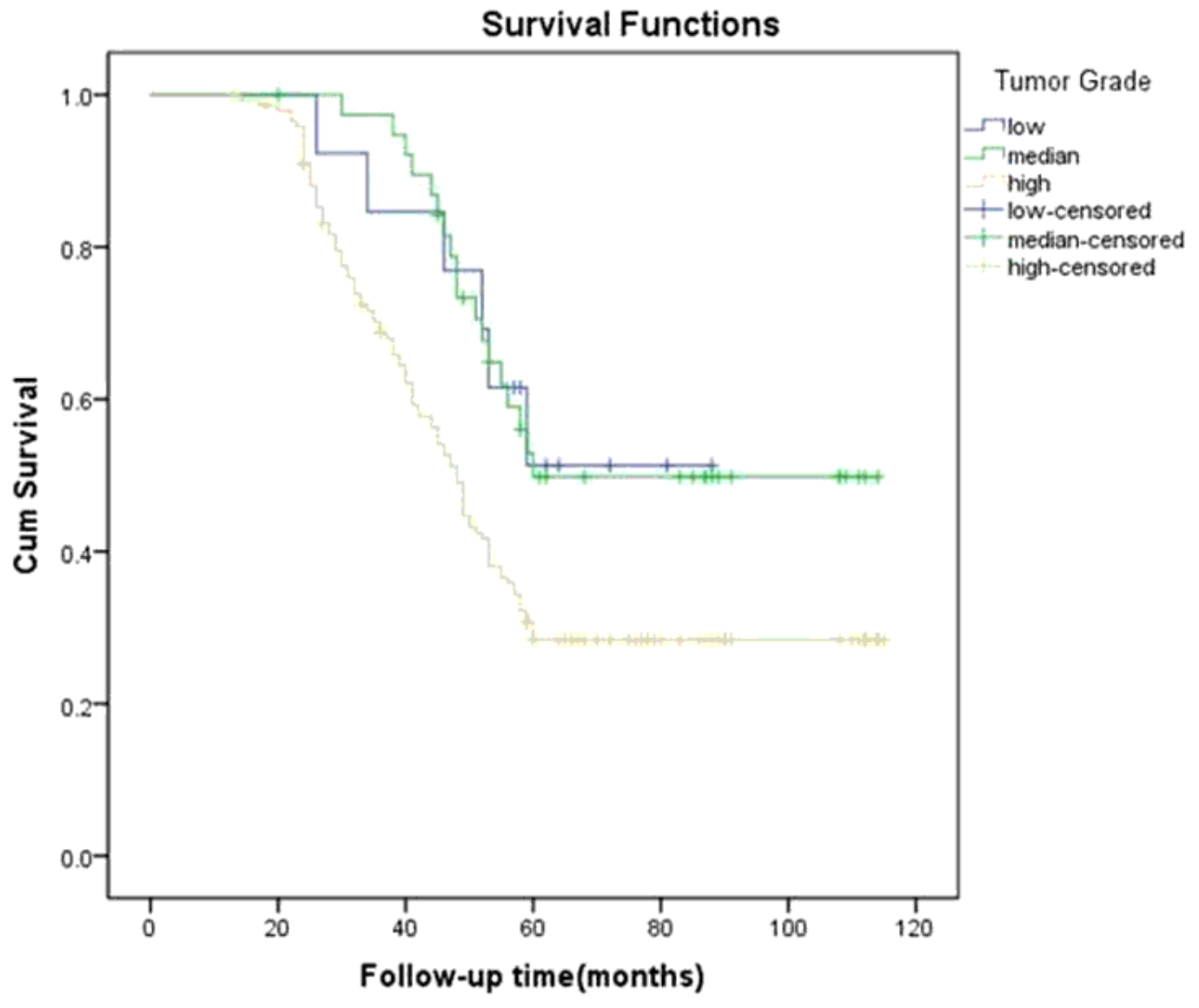

Figure 5

Disease stage, lymphovascular invasion and tumor grade as three independent predictors of DSS. A. Disease-specific survival function in patients with different disease stage $(p<0.001)$. B. Disease-specific survival function in patients with different lymphovascular invasion $(p=0.001)$. C. Disease-specific survival function in patients with different tumor grade $(p=0.004)$. 
A

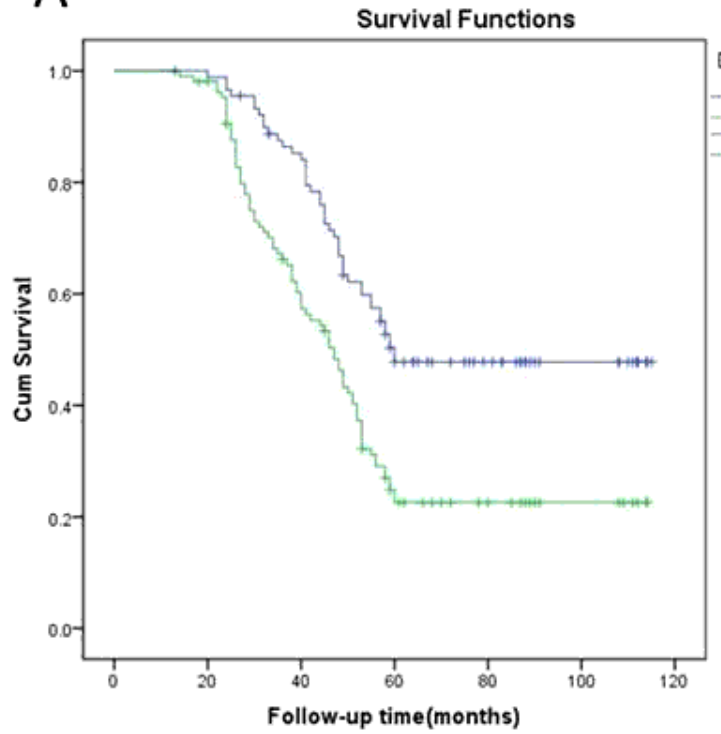

B

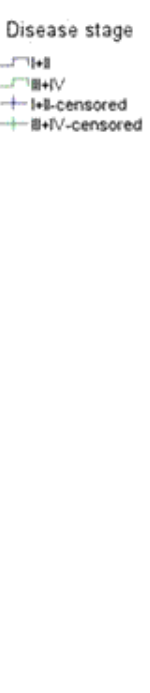

Survival Functions

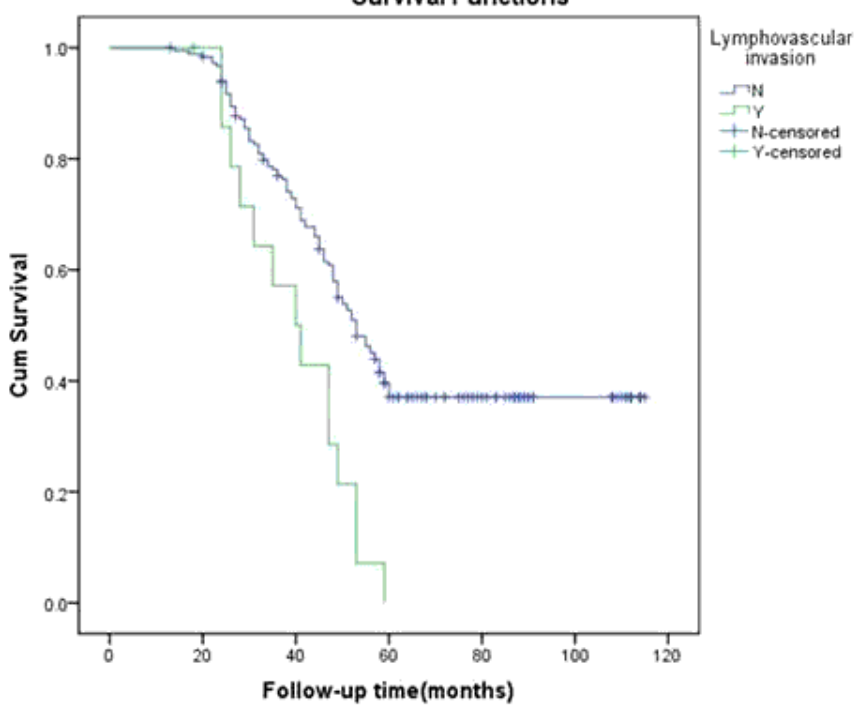

C

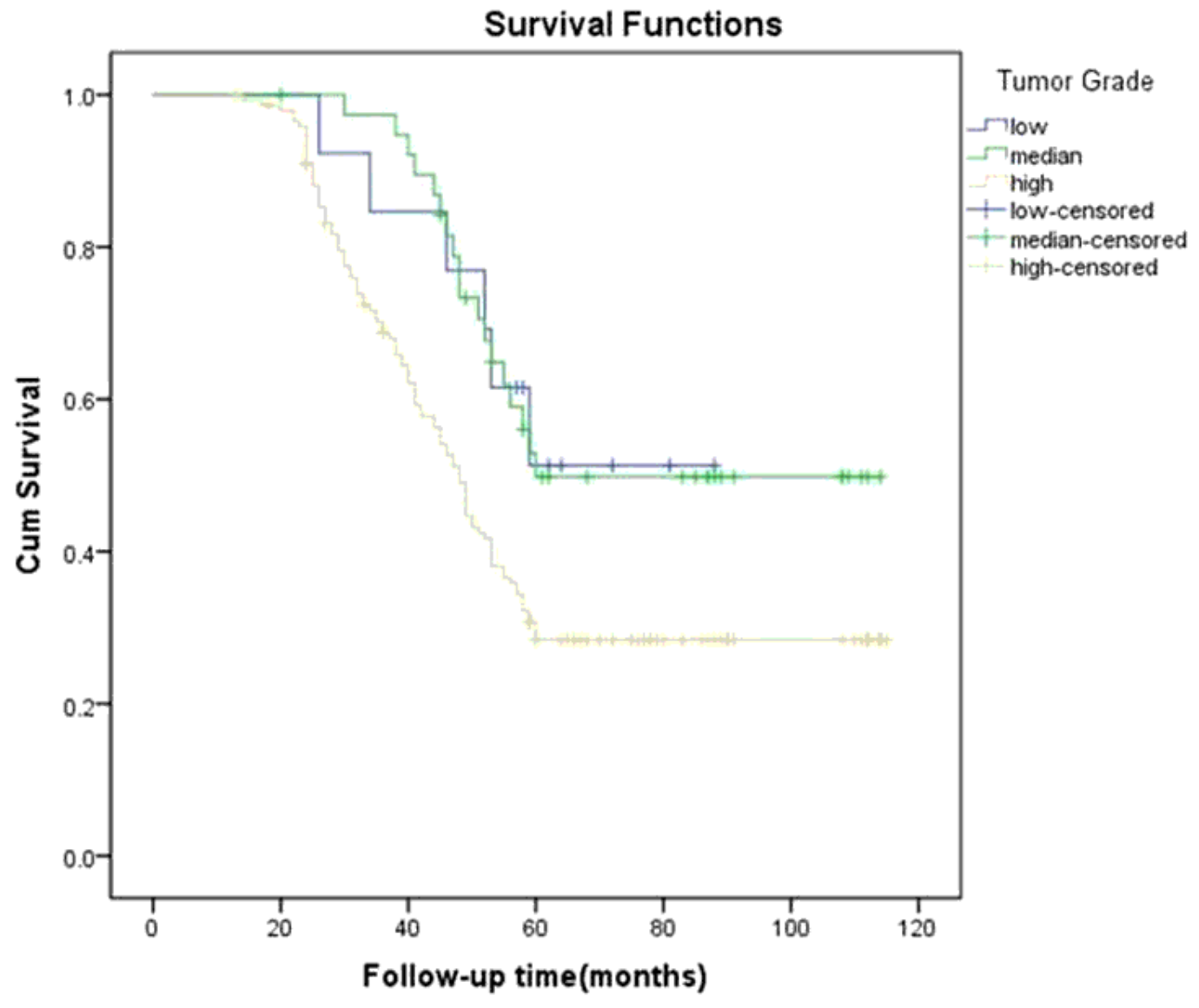

Figure 5

Disease stage, lymphovascular invasion and tumor grade as three independent predictors of DSS. A. Disease-specific survival function in patients with different disease stage $(p<0.001)$. B. Disease-specific survival function in patients with different lymphovascular invasion $(p=0.001)$. C. Disease-specific survival function in patients with different tumor grade $(p=0.004)$. 
A

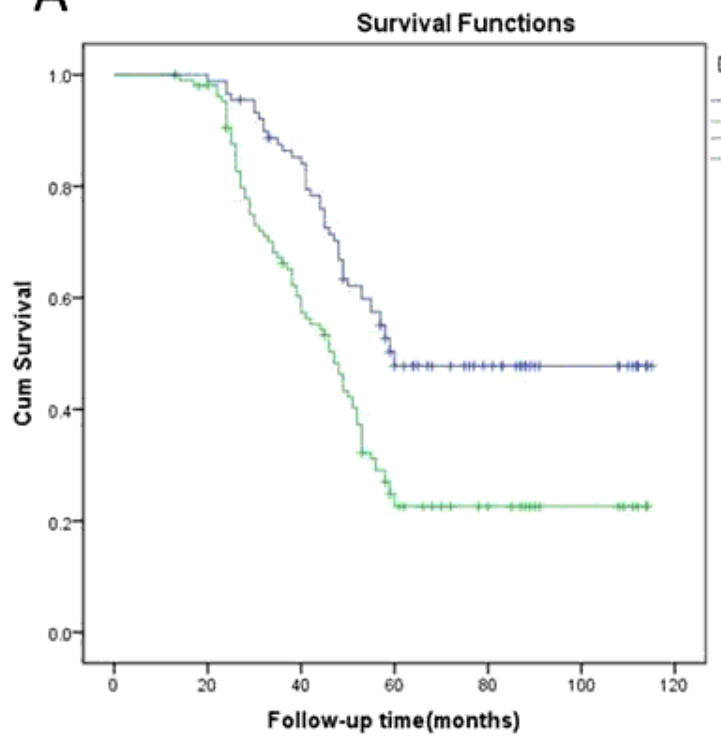

B

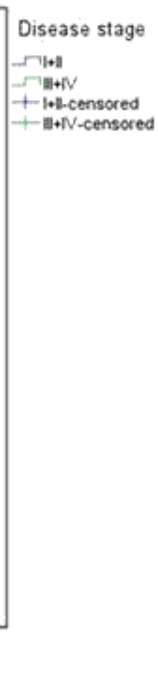

Survival Functions

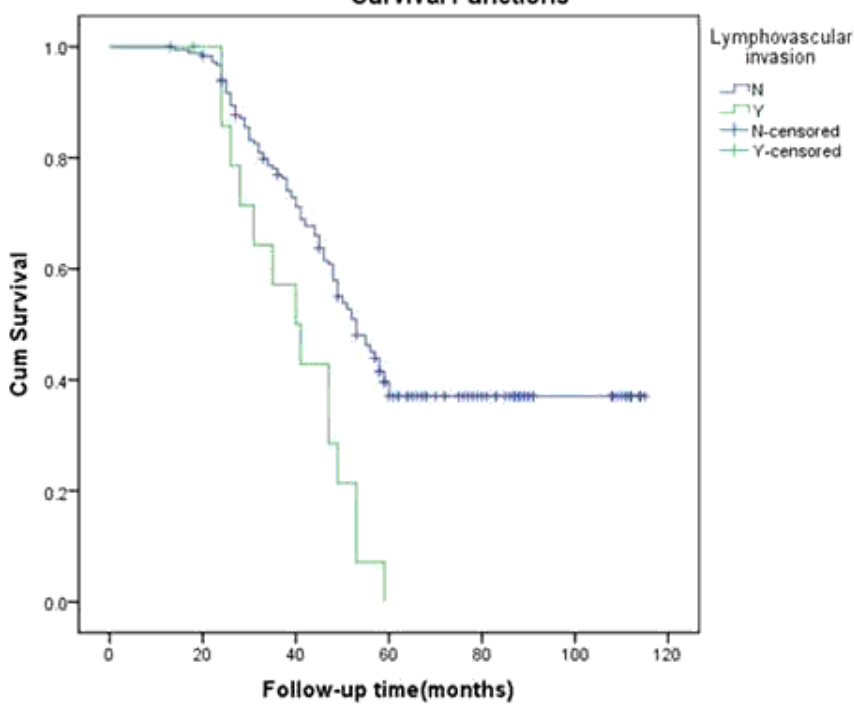

C

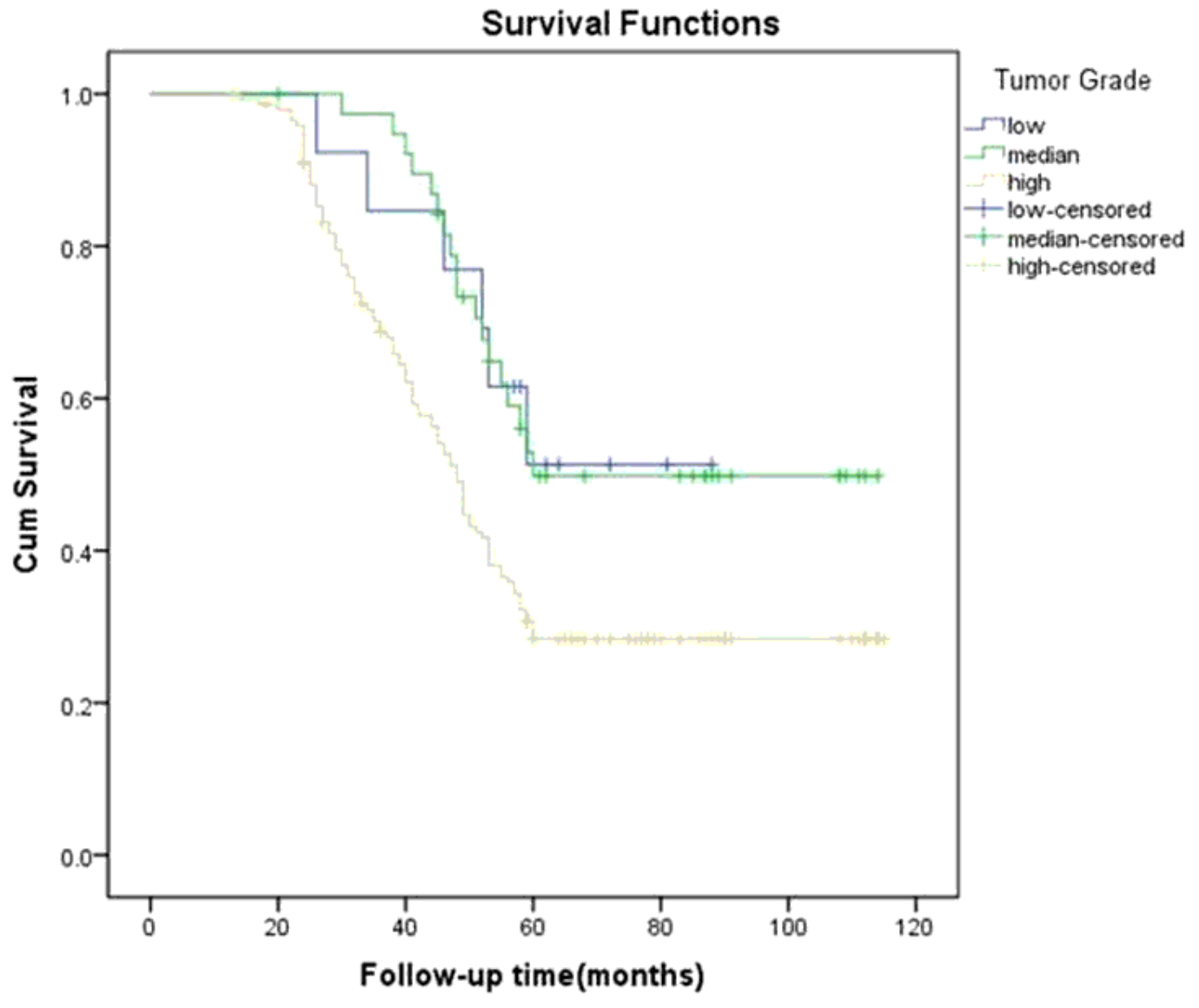

Figure 6

Disease stage, lymphovascular invasion and tumor grade as three independent predictors of DSS. A. Disease-specific survival function in patients with different disease stage $(p<0.001)$. B. Disease-specific survival function in patients with different lymphovascular invasion $(p=0.001)$. C. Disease-specific survival function in patients with different tumor grade $(p=0.004)$. 